\title{
Symplectic capacities of Hermitian symmetric spaces of compact and noncompact type
}

\author{
Andrea Loi, Roberto Mossa and Fabio Zuddas
}

Inspired by the work of G. Lu [35] on pseudo symplectic capacities we obtain several results on the Gromov width and the HoferZehnder capacity of Hermitian symmetric spaces of compact type. Our results and proofs extend those obtained by $\mathrm{Lu}$ for complex Grassmannians to Hermitian symmetric spaces of compact type. We also compute the Gromov width and the Hofer-Zehnder capacity for Cartan domains and their products.

\section{Introduction}

Consider the open ball of radius $r$,

$$
B^{2 n}(r)=\left\{\begin{array}{l|l}
(x, y) \in \mathbb{R}^{2 n} \mid \sum_{j=1}^{n} x_{j}^{2}+y_{j}^{2}<r^{2}
\end{array}\right\}
$$

in the standard symplectic space $\left(\mathbb{R}^{2 n}, \omega_{0}\right)$, where $\omega_{0}=\sum_{j=1}^{n} d x_{j} \wedge d y_{j}$. The Gromov width of a $2 n$-dimensional symplectic manifold $(M, \omega)$, introduced in [17], is defined as

$$
c_{G}(M, \omega)=\sup \left\{\pi r^{2} \mid B^{2 n}(r) \text { symplectically embeds into }(M, \omega)\right\} .
$$

By Darboux's theorem $c_{G}(M, \omega)$ is a positive number or $\infty$. Computations and estimates of the Gromov width for various examples can be found in [3], 4], [5], 17], [17, [22], [23], [28], [35], [36], 337, [38, [46], [51].

Gromov's width is an example of symplectic capacity introduced in [20] (see also [21]). A map $c$ from the class $\mathcal{C}(2 n)$ of all symplectic manifolds

The first two authors were supported by Prin 2010/11 - Varietà reali e complesse: geometria, topologia e analisi armonica - Italy. 
of dimension $2 n$ to $[0,+\infty]$ is called a symplectic capacity if it satisfies the following conditions:

(monotonicity) if there exists a symplectic embedding $\left(M_{1}, \omega_{1}\right) \rightarrow\left(M_{2}\right.$, $\left.\omega_{2}\right)$ then $c\left(M_{1}, \omega_{1}\right) \leq c\left(M_{2}, \omega_{2}\right)$;

(conformality) $c(M, \lambda \omega)=|\lambda| c(M, \omega)$, for every $\lambda \in \mathbb{R} \backslash\{0\}$;

(nontriviality) $c\left(B^{2 n}(1), \omega_{0}\right)=\pi=c\left(Z^{2 n}(1), \omega_{0}\right)$.

Here $B^{2 n}(1)$ and $Z^{2 n}(1)$ are the open unit ball and the open cylinder in the standard $\left(\mathbb{R}^{2 n}, \omega_{0}\right)$, i.e.

$$
Z^{2 n}(r)=\left\{(x, y) \in \mathbb{R}^{2 n} \mid x_{1}^{2}+y_{1}^{2}<r^{2}\right\}
$$

Note that the monotonicity property implies that $c$ is a symplectic invariant. The existence of a capacity is not a trivial matter. It is easily seen that the Gromov width is the smallest symplectic capacity, i.e. $c_{G}(M, \omega) \leq c(M, \omega)$ for any capacity $c$. Note that the nontriviality property for $c_{G}$ comes from the celebrated Gromov's nonsqueezing theorem according to which the existence of a symplectic embedding of $B^{2 n}(r)$ into $Z^{2 n}(R)$ implies $r \leq R$. Actually it is easily seen that the existence of any capacity implies Gromov's nonsqueezing theorem. H. Hofer and E. Zehnder [20] prove the existence of a capacity, denoted by $c_{H Z}$ which is important in many respects, for example it plays an important role in the study of Hofer geometry on the group of symplectomorphisms of a symplectic manifold and in establishing the existence of closed characteristics on or near an energy surface. To compute or estimate $c_{H Z}$ is rather difficult even for closed symplectic manifolds. So far the only known examples are closed surfaces where $c_{H Z}$ is just the area [48, and complex projective spaces and their products. H. Hofer and C. Viterbo [19] proved that $c_{H Z}\left(\mathbb{C P}^{n}, \omega_{F S}\right)=\pi$ which has been extended by G. Lu to the product of projective spaces (see Theorem 1.21 in 35] or (10) below). Lu's ingenious idea was that of defining and introducing the concept of pseudo symplectic capacity, more flexible than that of symplectic capacity, and studying its link with Gromov-Witten invariants (see Section 3 below). This allows him to obtain several valuable results, e.g. the Gromov width of Grassmannians and their products and a lower bound for the Hofer-Zehnder capacity for the product of any closed symplectic manifold with a Grassmannian. One of the aims of the present paper is to extend Lu's results to the case of Hermitian symmetric spaces of compact type.

Notation: From now on we shall use the shortening HSSCT to denote a Hermitian symmetric space of compact type. Further, throughout the paper 
we shall denote by $\omega_{F S}$ the canonical symplectic (Kähler) form on an irreducible HSSCT normalized so that $\omega_{F S}(B) \in\{-\pi, \pi\}$ when $B$ is a generator of $H_{2}(M, \mathbb{Z})$, and by $A$ the generator for which $\omega_{F S}(A)=\pi$.

Moreover, we compute the Gromov width and Hofer-Zehnder capacity of Cartan's domains and their products. In the next section we give a description of our results and the ideas of their proofs.

\section{Statements of the main results}

The following three theorems state our results about the Gromov width and the Hofer-Zehnder capacity of HSSCT.

Theorem 1. Let $\left(M, \omega_{F S}\right)$ be an irreducible HSSCT. Then

$$
c_{G}\left(M, \omega_{F S}\right)=\pi
$$

Theorem 2. Let $\left(M_{i}, \omega_{F S}^{i}\right), i=1, \ldots, r$, be irreducible HSSCT of complex dimension $n_{i}$ endowed with the canonical symplectic (Kähler) forms $\omega_{F S}^{i}$ normalized as above. Then

$$
c_{G}\left(M_{1} \times \cdots \times M_{r}, \omega_{F S}^{1} \oplus \cdots \oplus \omega_{F S}^{r}\right)=\pi .
$$

Moreover, if $a_{1}, \ldots, a_{r}$ are nonzero constants, then

$$
c_{G}\left(M_{1} \times \cdots \times M_{r}, a_{1} \omega_{F S}^{1} \oplus \cdots \oplus a_{r} \omega_{F S}^{r}\right) \leq \min \left\{\left|a_{1}\right|, \ldots,\left|a_{r}\right|\right\} \pi
$$

and

$$
c_{H Z}\left(M_{1} \times \cdots \times M_{r}, a_{1} \omega_{F S}^{1} \oplus \cdots \oplus a_{r} \omega_{F S}^{r}\right) \geq\left(\left|a_{1}\right|+\cdots+\left|a_{r}\right|\right) \pi .
$$

Theorem 3. Let $\left(M, \omega_{F S}\right)$ be an irreducible $\operatorname{HSSCT}$ and $(N, \omega)$ be any closed symplectic manifold. Then, for any nonzero real number a,

$$
c_{G}\left(N \times M, \omega \oplus a \omega_{F S}\right) \leq|a| \pi .
$$

Formulas (4) and (5) extend Theorem 1.15 and formula (22) in [35] respectively (valid for the Grassmannians) to the case of HSSCT. The lower bounds $c_{G}\left(M, \omega_{F S}\right) \geq \pi$ in Theorem 1 and

$$
c_{G}\left(M_{1} \times \cdots \times M_{r}, \omega_{F S}^{1} \oplus \cdots \oplus \omega_{F S}^{r}\right) \geq \pi
$$

in Theorem 2 are obtained by using the results in [11] which imply the existence of a symplectic embedding of the noncompact dual $\left(\Omega, \omega_{0}\right)$ of $\left(M, \omega_{F S}\right)$ 
into $\left(M, \omega_{F S}\right)$ (where $\omega_{0}$ is the standard symplectic form of $\Omega \subset \mathbb{C}^{n}$, being $n$ the complex dimension of $M)$ and by the existence of a symplectic embedding of $B^{2 n}(1)$ into $\left(\Omega, \omega_{0}\right)$ (see Sections 4 and 5 below for details). The upper bounds $c_{G}\left(M, \omega_{F S}\right) \leq \pi$ and

$$
c_{G}\left(M_{1} \times \cdots \times M_{r}, \omega_{F S}^{1} \oplus \cdots \oplus \omega_{F S}^{r}\right) \leq \pi
$$

are obtained by the use of Lu's pseudo symplectic capacities and their estimation in terms of Gromov-Witten invariants. The key ingredient to obtain these upper bounds is the non vanishing of some genus-zero three-points Gromov-Witten invariants (cfr. Lemma 16 in Section 6 below). Inequality (6), which extends (21) in [35] to HSSCT, is a consequence of (8) in Theorem 3, which in turn extends [35, Corollary 1.31].

When $M_{j}=\mathbb{C P}^{1}$ for all $j=1, \ldots, r$, inequality $(6)$ is indeed an equality, i.e.

$$
c_{G}\left(\mathbb{C P}^{1} \times \cdots \times \mathbb{C P}^{1}, a_{1} \omega_{F S} \oplus \cdots \oplus a_{r} \omega_{F S}\right)=\min \left\{\left|a_{1}\right|, \ldots,\left|a_{r}\right|\right\} \pi .
$$

(see [40, Example 12.5] for a proof). We do not know the exact value of

$$
c_{G}\left(\mathbb{C P}^{n_{1}} \times \cdots \times \mathbb{C P}^{n_{r}}, a_{1} \omega_{F S}^{1} \oplus \cdots \oplus a_{r} \omega_{F S}^{r}\right)
$$

if $n_{i}>1$ or $a_{j} \neq 1$ for some $i=1, \ldots, r$ or $j=1, \ldots, r$.

When the $M_{j}$ 's are projective spaces it was proved in Theorem 1.21 of [35] that the inequality (7) is an equality, namely

$$
c_{H Z}\left(\mathbb{C P}^{n_{1}} \times \cdots \times \mathbb{C P}^{n_{r}}, a_{1} \omega_{F S}^{1} \oplus \cdots \oplus a_{r} \omega_{F S}^{r}\right)=\left(\left|a_{1}\right|+\cdots+\left|a_{r}\right|\right) \pi
$$

In fact, Lu [35] was able to prove that

$$
c_{H Z}\left(\mathbb{C P}^{n_{1}} \times \cdots \times \mathbb{C P}^{n_{r}}, a_{1} \omega_{F S}^{1} \oplus \cdots \oplus a_{r} \omega_{F S}^{r}\right) \leq\left(\left|a_{1}\right|+\cdots+\left|a_{r}\right|\right) \pi
$$

which, combined with (7), yields (10). To the authors' best knowledge no upper bound of $c_{H Z}\left(M, \omega_{F S}\right)$ is known for $\operatorname{HSSCT}\left(M, \omega_{F S}\right)$, even for the case of the complex Grassmannians (different from the projective space). The idea's of Lu's proof of the upper bound (11) is sketched in Remark 23 . where we also explain why his argument cannot be used to achieve a similar upper bound for HSSCT.

We summarize our knowledge on Gromov width and Hofer-Zehnder capacity of Cartan domains in the following two theorems. 
Theorem 4. Let $\left(\Omega, \omega_{0}\right)$ be a Cartan domain. Then

$$
c_{G}\left(\Omega, \omega_{0}\right)=\pi
$$

and

$$
c_{H Z}\left(\Omega, \omega_{0}\right)=\pi
$$

Moreover, if $\Omega_{i} \subset \mathbb{C}^{n_{i}}, i=1, \ldots, r$ are Cartan domains of complex dimension $n_{i}$ equipped with the standard symplectic form $\omega_{0}^{i}$ of $\mathbb{R}^{2 n_{i}}=\mathbb{C}^{n_{i}}$, then

$$
c_{G}\left(\Omega_{1} \times \cdots \times \Omega_{r}, \omega_{0}^{1} \oplus \cdots \oplus \omega_{0}^{r}\right)=\pi .
$$

If $a_{1}, \ldots, a_{r}$ are nonzero constants, then

$$
c_{G}\left(\Omega_{1} \times \cdots \times \Omega_{r}, a_{1} \omega_{0}^{1} \oplus \cdots \oplus a_{r} \omega_{0}^{r}\right) \leq \min \left\{\left|a_{1}\right|, \ldots,\left|a_{r}\right|\right\} \pi
$$

Theorem 5. Let $\left(\Omega, \omega_{0}\right)$ be a Cartan domain and let $(N, \omega)$ be any closed symplectic manifold. Then

$$
c_{H Z}\left(N \times \Omega, \omega \oplus \omega_{0}\right)=\pi .
$$

The proof of Theorem 4 is based (together with the inclusion $B^{2 n}(1) \subset$ $\left.\left(\Omega, \omega_{0}\right)\right)$ on the fact that any $n$-dimensional Cartan domain $\left(\Omega, \omega_{0}\right)$ symplectically embeds into the cylinder $\left(Z^{2 n}(1), \omega_{0}\right)$ (see Sections 4 and 5 for details). Our result extends to all Cartan domains, including the exceptional ones, the results in [36].

Remark 6. Notice that the Cartan domains in this paper are linearly equivalent to the Cartan domains in the classical terminology (see Section 5 below). Thus, if one compares our results with those in [36] one has to pay attention to the multiplicative constants involved. For example, the Gromov width of $\left(R_{I V}(4), \omega_{0}\right)$ (the fourth Cartan domain in the classical terminology) as computed in [36] turns out to be equal to $\frac{\pi}{2}$ and the corresponding Cartan domain $\Omega$ (in our terminology) is given by $\Omega=\sqrt{2} R_{I V}(4)$, in accordance with our Theorem 4 .

The organization of the paper is as follows. In Section 3 we summarize the above mentioned Lu's work and some of his results needed in this paper. In Section 4 we briefly recall some tools on Hermitian positive Jordan triple systems which will be used in Section 5 to construct the above mentioned 
embeddings a) of a Cartan domain into its compact dual, b) of the unit ball into a Cartan domain and c) of a Cartan domain into the unitary cylinder. Moreover, in Subsection 5.1 we show how these symplectic embeddings could be used to estimate the minimal number of Darboux charts needed to cover a HSSCT. Finally, Section 6 is dedicated to the (conclusion of the) proofs of our theorems.

Acknowledgments. The authors are indebted to Guy Roos for suggesting us the idea of the construction of the symplectic embedding of a Cartan domain into the unitary cylinder via the use of Hermitian positive Jordan triple systems. The authors would like also to thank Dietmar Salamon for useful remarks on Gromov-Witten invariants and Giuseppina D'Ambra for several useful remarks which helped us to improve the exposition.

\section{Lu's pseudo symplectic capacities and Gromov-Witten invariants}

G. Lu [35] defines the concept of pseudo symplectic capacity by weakening the requirements for a symplectic capacity (see the Introduction) in such a way that this new concept depends on the homology classes of the symplectic manifold in question (for more details the reader is referred to [35]). More precisely, if one denotes by $\mathcal{C}(2 n, k)$ the set of all tuples $\left(M, \omega ; \alpha_{1}, \ldots, \alpha_{k}\right)$ consisting of a $2 n$-dimensional connected symplectic manifold $(M, \omega)$ and $k$ nonzero homology classes $\alpha_{i} \in H_{*}(M ; \mathbb{Q}), i=1, \ldots, k$, a map $c^{(k)}$ from $\mathcal{C}(2 n, k)$ to $[0,+\infty]$ is called a $k$-pseudo symplectic capacity if it satisfies the following properties:

(pseudo monotonicity) if there exists a symplectic embedding $\varphi$ : $\left(M_{1}, \omega_{1}\right) \rightarrow\left(M_{2}, \omega_{2}\right)$ then, for any $\alpha_{i} \in H_{*}\left(M_{1} ; \mathbb{Q}\right), i=1, \ldots, k$,

$$
c^{(k)}\left(M_{1}, \omega_{1} ; \alpha_{1}, \ldots, \alpha_{k}\right) \leq c^{(k)}\left(M_{2}, \omega_{2} ; \varphi_{*}\left(\alpha_{1}\right), \ldots, \varphi_{*}\left(\alpha_{k}\right)\right)
$$

(conformality) $c^{(k)}\left(M, \lambda \omega ; \alpha_{1}, \ldots, \alpha_{k}\right)=|\lambda| c^{(k)}\left(M, \omega ; \alpha_{1}, \ldots, \alpha_{k}\right)$, for every $\lambda \in \mathbb{R} \backslash\{0\}$ and all homology classes $\alpha_{i} \in H_{*}(M ; \mathbb{Q}) \backslash\{0\}, i=1, \ldots, k$;

(nontriviality) $c\left(B^{2 n}(1), \omega_{0} ; p t, \ldots, p t\right)=\pi=c\left(Z^{2 n}(1), \omega_{0} ; p t, \ldots, p t\right)$, where $p t$ denotes the homology class of a point.

Note that if $k>1$ a $(k-1)$-pseudo symplectic capacity is defined by

$$
c^{(k-1)}\left(M, \omega ; \alpha_{1}, \ldots, \alpha_{k-1}\right):=c^{(k)}\left(M, \omega ; p t, \alpha_{1}, \ldots, \alpha_{k-1}\right)
$$


and any $c^{(k)}$ induces a true symplectic capacity

$$
c^{(0)}(M, \omega):=c^{(k)}(M, \omega ; p t, \ldots, p t) .
$$

Observe also that (unlike symplectic capacities) pseudo symplectic capacities do not define symplectic invariants.

In [35] G. Lu was able to construct two 2-pseudo symplectic capacities denoted by $C_{H Z}^{(2)}\left(M, \omega ; \alpha_{1}, \alpha_{2}\right)$ and $C_{H Z}^{(2 o)}\left(M, \omega ; \alpha_{1}, \alpha_{2}\right)$ respectively (see Definition 1.3 and Theorem 1.5 in [35]), where $\alpha_{1}$ and $\alpha_{2}$ are homology classes ${ }^{1}$ in $H_{*}(M ; \mathbb{Q})$. The $C_{H Z}^{(2)}$ and $C_{H Z}^{(2 o)}$ are called by Lu pseudo symplectic capacities of Hofer-Zehnder type.

Denote by

$$
C_{H Z}(M, \omega):=C_{H Z}^{(2)}(M, \omega ; p t, p t)
$$

(resp. $\left.C_{H Z}^{o}(M, \omega):=C_{H Z}^{(2 o)}(M, \omega ; p t, p t)\right)$ the corresponding true symplectic capacities associated to Lu's pseudo symplectic capacities. The next lemma summarizes some properties of the concepts involved so far.

Lemma 7. Let $(M, \omega)$ be any symplectic manifold. Then, for arbitrary homology classes $\alpha_{1}, \alpha_{2} \in H_{*}(M ; \mathbb{Q})$ and for a nonzero homology class $\alpha$, with $\operatorname{dim} \alpha \leq \operatorname{dim} M-1$, the following inequalities hold true:

$$
\begin{aligned}
C_{H Z}^{(2)}\left(M, \omega ; \alpha_{1}, \alpha_{2}\right) & \leq C_{H Z}^{(2 o)}\left(M, \omega ; \alpha_{1}, \alpha_{2}\right) \\
C_{H Z}^{(2)}\left(M, \omega ; \alpha_{1}, \alpha_{2}\right) & \leq C_{H Z}(M, \omega) \leq c_{H Z}(M, \omega) \\
C_{H Z}^{(2 o)}\left(M, \omega ; \alpha_{1}, \alpha_{2}\right) & \leq C_{H Z}^{o}(M, \omega) \leq c_{H Z}^{o}(M, \omega) \\
c_{G}(M, \omega) & \leq C_{H Z}^{(2)}(M, \omega ; p t, \alpha),
\end{aligned}
$$

where $c_{H Z}^{o}(M, \omega)$ is the $\pi_{1}$-sensitive Hofer-Zehnder capacity introduced in [47] (and independently in [34]), $c_{H Z}(M, \omega)$ is the Hofer-Zehnder capacity and $c_{G}(M, \omega)$ is the Gromov width of $(M, \omega)$. Furthermore, if $M$ is closed then

$$
C_{H Z}(M, \omega)=c_{H Z}(M, \omega)
$$

and

$$
C_{H Z}^{o}(M, \omega)=c_{H Z}^{o}(M, \omega)
$$

\footnotetext{
${ }^{1}$ In the notations of 35 the generic classes $\alpha_{1}$ (resp. $\left.\alpha_{2}\right)$ are called $\alpha_{0}$ (resp. $\left.\alpha_{\infty}\right)$. The reason for this notation comes from the concept of hypersurface $S \subset M$ separating the homology classes $\alpha_{0}$ and $\alpha_{\infty}$ (see Definition 1.3 and the $\left(\alpha_{0}, \alpha_{\infty}\right)$ Weinstein conjecture at p.6 of [35]).
} 
Proof. See Lemma 1.4 and (12) in [35].

Remark 8. The last two equalities together with 17) imply that for a closed symplectic manifold $(M, \omega)$

$$
c_{H Z}(M, \omega) \leq c_{H Z}^{o}(M, \omega) .
$$

It follows that inequality (7) in Theorem 2 holds true also when we replace $c_{H Z}$ with $c_{H Z}^{o}$.

When the symplectic manifold $M$ is closed the pseudo symplectic capacities $C_{H Z}^{(2)}\left(M, \omega ; \alpha_{1}, \alpha_{2}\right)$ and $C_{H Z}^{(2 o)}\left(M, \omega ; \alpha_{1}, \alpha_{2}\right)$ can be estimated by other two pseudo symplectic capacities $G W\left(M, \omega ; \alpha_{1}, \alpha_{2}\right) \quad$ and $G W_{0}\left(M, \omega ; \alpha_{1}, \alpha_{2}\right)$. These $G W$ and $G W_{0}$ are defined in terms of Liu-Tian type Gromov-Witten invariants as follows. Let $B \in H_{2}(M, \mathbb{Z})$ : the Liu-Tian type Gromov-Witten invariant of genus $g$ and with $k$ marked points is a homomorphism

$$
\Psi_{B, g, k}^{M}: H_{*}\left(\overline{\mathcal{M}}_{g, k} ; \mathbb{Q}\right) \times H_{*}(M ; \mathbb{Q})^{k} \rightarrow \mathbb{Q}, 2 g+k \geq 3
$$

where $\overline{\mathcal{M}}_{g, k}$ is the space of isomorphism classes of genus $g$ stable curves with $k$ marked points. When there is no risk of confusion, we will omit the superscript $M$ in $\Psi_{B, g, k}^{M}$. Roughly speaking, one can think of $\Psi_{B, g, k}^{M}\left(\mathcal{C} ; \alpha_{1}, \ldots, \alpha_{k}\right)$ as counting, for suitable generic $\omega$-tame almost complex structure $J$ on $M$, the number of $J$-holomorphic curves of genus $g$ representing $B$, with $k$ marked points $p_{i}$ which pass through cycles $X_{i}$ representing $\alpha_{i}$, and such that the image of the curve belongs to a cycle representing $\mathcal{C}$ (for details the reader is referred to the Appendix in [35] and references therein).

In fact, several different constructions of Gromov-Witten invariants appear in the literature and the question whether they agree is not trivial (see [35] and also Chapter 7 in [39]). The Gromov-Witten invariants described in the book of D. McDuff and D. Salamon [39] are the most commonly used: these are homomorphisms

$$
\Psi_{B, g, m+2}: H_{*}(M ; \mathbb{Q})^{m+2} \rightarrow \mathbb{Q}, m \geq 1
$$

which play an important role in the proofs of this paper. The conditions under which these invariants agree with the ones considered by Lu are given in Lemma 10 below.

Let $\alpha_{1}, \alpha_{2} \in H_{*}(M, \mathbb{Q})$. Following [35], one defines $G W_{g}\left(M, \omega ; \alpha_{1}, \alpha_{2}\right) \in(0$, $+\infty]$ as the infimum of the $\omega$-areas $\omega(B)$ of the homology classes $B \in$ 
$H_{2}(M, \mathbb{Z})$ for which the Liu-Tian Gromov-Witten invariant $\Psi_{B, g, m+2}(C$; $\left.\alpha_{1}, \alpha_{2}, \beta_{1}, \ldots, \beta_{m}\right) \neq 0$ for some homology classes $\beta_{1}, \ldots, \beta_{m} \in H_{*}(M, \mathbb{Q})$ and $C \in H_{*}\left(\overline{\mathcal{M}}_{g, m+2} ; \mathbb{Q}\right)$ and integer $m \geq 1$ (we use the convention $\inf \emptyset=$ $+\infty)$. The positivity of $G W_{g}$ reflects the fact that $\Psi_{B, g, m+2}=0$ if $\omega(B)<0$ (see, for example, Section 7.5 in [39]). Set

$$
G W\left(M, \omega ; \alpha_{1}, \alpha_{2}\right):=\inf \left\{G W_{g}\left(M, \omega ; \alpha_{1}, \alpha_{2}\right) \mid g \geq 0\right\} \in[0,+\infty] .
$$

Lemma 9. Let $(M, \omega)$ be a closed symplectic manifold. Then

$$
0 \leq G W\left(M, \omega ; \alpha_{1}, \alpha_{2}\right) \leq G W_{0}\left(M, \omega ; \alpha_{1}, \alpha_{2}\right)
$$

Moreover $G W\left(M, \omega ; \alpha_{1}, \alpha_{2}\right)$ and $G W_{0}\left(M, \omega ; \alpha_{1}, \alpha_{2}\right)$ are pseudo symplectic capacities and, if $\operatorname{dim} M \geq 4$ then, for nonzero homology classes $\alpha_{1}, \alpha_{2}$, we have

$$
\begin{aligned}
& C_{H Z}^{(2)}\left(M, \omega ; \alpha_{1}, \alpha_{2}\right) \leq G W\left(M, \omega ; \alpha_{1}, \alpha_{2}\right) \\
& C_{H Z}^{(2 o)}\left(M, \omega ; \alpha_{1}, \alpha_{2}\right) \leq G W_{0}\left(M, \omega ; \alpha_{1}, \alpha_{2}\right) .
\end{aligned}
$$

In particular, for every nonzero homology class $\alpha \in H_{*}(M, \mathbb{Q})$,

$$
\begin{aligned}
& C_{H Z}^{(2)}(M, \omega ; p t, \alpha) \leq G W(M, \omega ; p t, \alpha) \\
& C_{H Z}^{(2 o)}(M, \omega ; p t, \alpha) \leq G W_{0}(M, \omega ; p t, \alpha) .
\end{aligned}
$$

Proof. See Theorems 1.10 and 1.13 in [35].

We end this section with the following lemmata fundamental for the proof of our results. Recall that a closed symplectic manifold is monotone if there exists a number $\lambda>0$ such that $\omega(B)=\lambda c_{1}(B)$ for $B$ spherical (a homology class is called spherical if it is in the image of the Hurewicz homomorphism $\pi_{2}(M) \rightarrow H_{2}(M, \mathbb{Z})$ ). Further, a homology class $B \in H_{2}(M, \mathbb{Z})$ is indecomposable if it cannot be decomposed as a sum $B=B_{1}+\cdots+B_{k}, k \geq 2$, of classes which are spherical and satisfy $\omega\left(B_{i}\right)>0$ for $i=1, \ldots, k$.

Lemma 10. Let $(M, \omega)$ be a closed monotone symplectic manifold. Let $B \in H_{2}(M, \mathbb{Z})$ be an indecomposable spherical class, let pt denote the class of a point in $H_{*}\left(\overline{\mathcal{M}}_{g, m+2} ; \mathbb{Q}\right)$ and let $\alpha_{i} \in H_{*}(M, \mathbb{Z}), i=1,2,3$. Then the Liu-Tian Gromov-Witten invariant $\Psi_{B, 0,3}\left(p t ; \alpha_{1}, \alpha_{2}, \alpha_{3}\right)$ agrees with the Gromov-Witten invariant $\Psi_{B, 0,3}\left(\alpha_{1}, \alpha_{2}, \alpha_{3}\right)$.

Proof. See [35, Proposition 7.6]. 
Lemma 11. Let $\left(N_{1}, \omega_{1}\right)$ and $\left(N_{2}, \omega_{2}\right)$ be two closed symplectic manifolds. Then for every integer $k \geq 3$ and homology classes $A_{2} \in H_{2}\left(N_{2} ; \mathbb{Z}\right)$ and $\beta_{i} \in$ $H_{*}\left(N_{2} ; \mathbb{Z}\right), i=1, \ldots, k$,

$$
\Psi_{0 \oplus A_{2}, 0, k}^{N_{1} \times N_{2}}\left(p t ;\left[N_{1}\right] \otimes \beta_{1}, \ldots,\left[N_{1}\right] \otimes \beta_{k-1}, p t \otimes \beta_{k}\right)=\Psi_{A_{2}, 0, k}^{N_{2}}\left(p t ; \beta_{1}, \ldots, \beta_{k}\right) .
$$

Proof. See [35, Proposition 7.4].

\section{Hermitian positive Jordan triple system}

We refer the reader to [43] (see also [33]) for more details on Hermitian symmetric spaces of noncompact type (HSSNT) and Hermitian positive Jordan triple systems (HPJTS).

Definitions and notations. A Hermitian Jordan triple system is a pair $(\mathcal{M},\{,\}$,$) , where \mathcal{M}$ is a complex vector space and $\{,$,$\} is a map$

$$
\begin{gathered}
\{,,\}: \mathcal{M} \times \mathcal{M} \times \mathcal{M} \rightarrow \mathcal{M} \\
(u, v, w) \mapsto\{u, v, w\}
\end{gathered}
$$

which is $\mathbb{C}$-bilinear and symmetric in $u$ and $w, \mathbb{C}$-antilinear in $v$ and such that the following Jordan identity holds:

$$
\begin{aligned}
& \{x, y,\{u, v, w\}\}-\{u, v,\{x, y, w\}\} \\
= & \{\{x, y, u\}, v, w\}-\{u,\{v, x, y\}, w\} .
\end{aligned}
$$

For $x, y, z \in \mathcal{M}$ consider the operators

$$
\begin{aligned}
T(x, y) z & =\{x, y, z\} \\
Q(x, z) y & =\{x, y, z\} \\
Q(x, x) & =2 Q(x) \\
B(x, y) & =\operatorname{id}_{\mathcal{M}}-T(x, y)+Q(x) Q(y) .
\end{aligned}
$$

The operator $B(x, y)$ is called the Bergman operator. A Hermitian Jordan triple system is called positive if the sesquilinear form

$$
(u \mid v)=\frac{1}{\gamma} \operatorname{tr} T(u, v)
$$

is a Hermitian product, where $\gamma$ is a positive constant called the genus of $(\mathcal{M},\{,\}$,$) .$ 
HSSNT associated to HPJTS. M. Koecher ([25, [26]) discovered that to every HPJTS $(\mathcal{M},\{,\}$,$) one can associate an Hermitian symmetric space of$ noncompact type, in its realization as circled ${ }^{2}$ bounded symmetric domain $\Omega_{\mathcal{M}}$ centered at the origin $0 \in \mathcal{M}$. More precisely, $\Omega_{\mathcal{M}}$ is defined as the connected component containing the origin of the set of all $u \in \mathcal{M}$ such that $B(u, u)$ is positive definite with respect to the Hermitian product (24).

HPJTS associated to HSSNT. The HPJTS $(\mathcal{M},\{,\}$,$) can be recovered$ from its associated HSSNT $\Omega_{\mathcal{M}}$ by defining $\mathcal{M}=T_{0} \Omega_{\mathcal{M}}$ (the tangent space to the origin of $\Omega_{\mathcal{M}}$ ) and

$$
\{u, v, w\}=-\frac{1}{2}\left(R_{0}(u, v) w+J_{0} R_{0}\left(u, J_{0} v\right) w\right)
$$

where $R_{0}$ (resp. $J_{0}$ ) is the curvature tensor of the Bergman metric (resp. the complex structure) of $\Omega_{\mathcal{M}}$ evaluated at the origin. The reader is referred to Proposition III.2.7 in [2] for details. To learn more on the correspondence between HPJTS and HSSNT we refer to p. 85 of Satake's book [45].

$0.3 \mathrm{~cm}$ Spectral decomposition. Let $(\mathcal{M},\{,\}$,$) be a HPJTS. An ele-$ ment $c \in \mathcal{M}$ is called tripotent if $\{c, c, c\}=2 c$. Two tripotents $c_{1}$ and $c_{2}$ are called (strongly) orthogonal if $T\left(c_{1}, c_{2}\right)=0$. Each element $v \in \mathcal{M}$ has a unique spectral decomposition

$$
v=\lambda_{1} c_{1}+\cdots+\lambda_{s} c_{s} \quad\left(\lambda_{1}>\cdots>\lambda_{s}>0\right)
$$

where $\left(c_{1}, \ldots, c_{s}\right)$ is a sequence of pairwise orthogonal (with respect to 24) tripotents and the $\lambda_{j}$ 's are real numbers called eigenvalues of $v$. The integer $s$ is called the rank of $v$ and is denoted by $\operatorname{rk}(v)$. The $\operatorname{rank}$ of $\mathcal{M}$ is the positive integer $r$ defined as $r=\max \{\operatorname{rk}(z) \mid z \in \mathcal{M}\}$. The elements $z \in \mathcal{M}$ such that $\operatorname{rk}(z)=r$ are called regular.

Let us denote by $\|v\|_{\max }$ the largest eigenvalue of $v$. Due to the convexity of $\Omega_{\mathcal{M}},\|v\|_{\max }$ is a norm on $\mathcal{M}$, called the spectral norm. The following proposition provides a description of the domain $\Omega_{\mathcal{M}}$ in terms of its spectral norm.

Proposition 12. Let $\Omega_{\mathcal{M}} \subset \mathcal{M}$ be the $H S S N T$ associated to $(\mathcal{M},\{,\}$,$) .$ Then

$$
\Omega_{\mathcal{M}}=\left\{v \mid\|v\|_{\max }<1\right\}
$$

Proof. See [33, Corollary 3.15].

\footnotetext{
${ }^{2}$ The domain $\Omega \subset \mathcal{M}$ is circled if $e^{i \theta} \cdot \Omega=\Omega$
} 


\section{Cartan domains, their compact duals and some symplectic embeddings}

Let $(\mathcal{M},\{,\}$,$) be a HPJTS and \Omega_{\mathcal{M}}$ be its associated HSSNT. Let $n$ be the complex dimension of $\mathcal{M}$. By fixing an orthonormal basis $\underline{e}=\left\{e_{1}, \ldots, e_{n}\right\}$ of $(\mathcal{M},(\cdot \mid \cdot))$ we get the identification

$$
\mathcal{M} \rightarrow \mathbb{C}^{n}, \quad v \stackrel{e}{\mapsto} z=\left(z_{1}, \ldots, z_{n}\right), \quad v=z_{1} e_{1}+\cdots+z_{n} e_{n},
$$

which induces an isometry between $(\mathcal{M},(\cdot \mid \cdot))$ and $\left(\mathbb{C}^{n}, h_{0}\right)$, where $h_{0}$ is the canonical Hermitian product on $\mathbb{C}^{n}$. Under the identification

$$
\left(z_{1}, \ldots, z_{n}\right)=\left(x_{1}, y_{1}, \ldots, x_{n}, y_{n}\right)
$$

between $\mathbb{C}^{n}$ and $\mathbb{R}^{2 n}$ we have $h_{0}=g_{0}+i \omega_{0}$, where $g_{0}=\sum_{j=1}^{n} d x_{j}^{2}+d y_{j}^{2}$ is the standard scalar product on $\mathbb{R}^{2 n}$ and $\omega_{0}$ is the canonical symplectic form $\omega_{0}=\sum_{j=1}^{n} d x_{j} \wedge d y_{j}$ on $\mathbb{C}^{n}=\mathbb{R}^{2 n}$. From now on we assume $\mathcal{M}$ to be simple, which is equivalent to the irreducibility of $\Omega_{\mathcal{M}}$. Then, under the previous identification, the HSSNT $\Omega_{\mathcal{M}}$ corresponds to a bounded symmetric domain $\Omega=\bar{e}\left(\Omega_{\mathcal{M}}\right) \subset \mathbb{C}^{n}$. The complex and Riemannian geometry of these domains is well-known (see, e.g. [24]). Indeed, each of these domains is linearly equivalent to a Cartan domain (see, e.g. [26, Chapter V] for a proof).

Terminology: In the present paper, with a slight abuse of terminology, the domain $\Omega=\bar{e}\left(\Omega_{\mathcal{M}}\right)$ has been called a Cartan domain.

We describe below some symplectic-geometric properties of Cartan domains and their compact duals which are needed in this paper (for the concept of compact dual see [18] or [11] and references therein).

Let $\Omega \subset \mathbb{C}^{n}$ be a Cartan domain and let $M$ be its compact dual. Then $M$ is an $n$-dimensional HSSCT. Denote by

$$
B W: M \rightarrow \mathbb{C} P^{N}
$$

the Borel-Weil (holomorphic) embedding. It is well-known (see e.g. [50]) that the pull-back $B W^{*} \omega_{\mathrm{FS}}$ of the Fubini-Study form $\omega_{\mathrm{FS}}$ of $\mathbb{C} P^{N}$ is a homogeneous Kähler-Einstein form on $M\left(\omega_{\mathrm{FS}}\right.$ is the Kähler form which, in the homogeneous coordinates $\left[z_{0}, \ldots, z_{N}\right]$ on $\mathbb{C} P^{N}$, is given by $\omega_{\mathrm{FS}}=$ $\frac{i}{2} \partial \bar{\partial} \log \left(\left|z_{0}\right|^{2}+\cdots+\left|z_{N}\right|^{2}\right)$ ). Here we denote (with a slight abuse of notation and terminology) by $\omega_{\mathrm{FS}}$ the form $B W^{*} \omega_{\mathrm{FS}}$ and call it the Fubini-Study form on $M$. The symplectic form $\omega_{F S}$ can be equivalently described as the 
symmetric or canonical form on $M$ normalized so that $\omega_{F S}(B)= \pm \pi$ when $B$ is a generator of $H_{2}(M, \mathbb{Z})$.

The domain $\left(\Omega, \omega_{0}\right)$ can be embedded into $\left(M, \omega_{\mathrm{FS}}\right)$.

Let $\left(\Omega, \omega_{0}\right), \Omega \subset \mathbb{C}^{n}$, be a Cartan domain equipped with the canonical symplectic form $\omega_{0}$ of $\mathbb{R}^{2 n}$ and let $\left(M, \omega_{F S}\right)$ be its compact dual. In [1] the first author in collaboration with A. J. Di Scala, by an use of HPJTS, construct an embedding

$$
\Phi_{\Omega}: \Omega \rightarrow M
$$

such that $\Phi_{\Omega}^{*} \omega_{F S}=\omega_{0}$.

Actually in [11] much more is proved. In particular it is shown that the embedding $\Phi_{\Omega}$ induces a global symplectomorphism

$$
\Phi_{\Omega}:\left(\Omega, \omega_{0}\right) \rightarrow\left(M \backslash \operatorname{Cut}_{0}(M), \omega_{F S}\right)
$$

where $\operatorname{Cut}_{0}(M)$ is the cut locus of $\left(M, \omega_{F S}\right)$ with respect to a fixed point $0 \in M$ (see [11, Theorem 1.1]). This diffeomorphism has been christened in [11] as a symplectic duality due to the fact that, amongst other properties, it also satisfies $\Phi_{\Omega}^{*} \omega_{0}=\omega_{\text {hyp }}$, where $\omega_{0}$ denotes the standard form on $\mathbb{C}^{n} \cong$ $M \backslash \operatorname{Cut}_{0}(M)$ and $\omega_{h y p}$ is the hyperbolic metric on $\Omega$ (see either [11] or [12] for details and also [9], [13], 30], 31], 29], [32] and [41] for the construction of explicit symplectic coordinates.

Remark 13. In [35, Lemma 4.1 in Section 4] it is shown the existence of a symplectic embedding

$$
\Phi_{\Omega_{I}[k, n]}: \Omega_{I}[k, n] \rightarrow G(k, n)
$$

from the first Cartan domain $\Omega_{I}[k, n] \subset \mathbb{C}^{k(n-k)}$ into its compact dual $G(k, n)$ (where $G(k, n)$ denotes the complex Grassmannian of $k$ dimensional subspaces of $\mathbb{C}^{n}$ ). Our result 28 extends Lu's results to all HSSCT.

The unitary ball $\left(B^{2 n}(1), \omega_{0}\right)$ can be embedded into $\left(\Omega, \omega_{0}\right)$.

Let $v=\lambda_{1} c_{1}+\cdots+\lambda_{r} c_{r}$ be the spectral decomposition of a regular point $v \in \Omega_{\mathcal{M}} \subset \mathcal{M}$, then the distance $\mathrm{d}_{0}(0, v)$ from the origin $0 \in \mathcal{M}$ to $v$ is given by

$$
\mathrm{d}_{0}(0, v)=(v \mid v)^{\frac{1}{2}}=\sqrt{\sum_{j=1}^{r} \lambda_{j}^{2}}
$$


(see [43, Proposition VI.3.6] for a proof). Since the set of regular points of $\mathcal{M}$ is dense ([43, Proposition IV.3.1]) we conclude, by (25) and by the identification $\Omega_{\mathcal{M}} \cong \Omega$ (induced by $\left.(\mathcal{M},(\cdot \mid \cdot)) \cong\left(\mathbb{C}^{n}, h_{0}\right)\right)$ that

$$
\left(B^{2 n}(1), \omega_{0}\right) \subset\left(\Omega, \omega_{0}\right) .
$$

Remark 14. The inclusion (31) has been obtained in [35, Lemma 4.2, Section 4] for the case of the first Cartan domain, namely $B^{2 k(n-k)}(1) \subset$ $\Omega_{I}[k, n]$ (see also [36] for the case of classical Cartan domains). Combining this with the symplectic embedding (29) Lu was able (see [35, Theorem 1.35]) to obtain the upper bound

$$
F\left(G(k, n), \omega_{F S}\right) \leq[n / k]
$$

where $F(N, \omega)$ denotes the Fefferman invariant of a closed symplectic manifold $(N, \omega)$, namely the largest integer $p$ for which there exists a symplectic packing by $p$ open unit balls, and $[n / k]$ is the largest integer less than or equal to $n / k$. The authors believe it is an intriguing problem to give a similar upper bound for all HSSCT by using the techniques of this paper.

The domain $\left(\Omega, \omega_{0}\right)$ can be embedded into $\left(Z^{2 n}(1), \omega_{0}\right)$.

Let $Z^{2 n}(1)=\left\{(x, y) \mid x_{1}^{2}+y_{1}^{2}<1\right\}$ be the unitary cylinder in $\mathbb{R}^{2 n}$. Let $v=\lambda_{1} c_{1}+\cdots+\lambda_{r} c_{r}$ be the spectral decomposition of a regular point $v \in$ $\Omega_{\mathcal{M}} \subset \mathcal{M}$. By $(30)$ and by the continuity of $\mathrm{d}_{0}$ (the distance function from the origin $0 \in \mathcal{M})$ we see that $\mathrm{d}_{0}\left(0, c_{1}\right)=1$. Set $c:=c_{1}$, by 33 , Corollary 4.8] $c \in \partial \Omega_{\mathcal{M}}$. Since $\Omega_{\mathcal{M}}$ is convex ([33, Corollary 4.7]), by the supporting hyperplane property there exists a real hyperplane $\pi$ of $\mathcal{M}$ through $c$ not intersecting $\Omega_{\mathcal{M}}$. Denote by $p=\bar{e}(c) \in \partial \Omega$ the image of the tripotent $c$ by the isometry 26 . Hence $p \in S^{2 n-1}$, where $S^{2 n-1}=\partial B^{2 n}(1)$ is the $(2 n-1)$ dimensional unit sphere centered at the origin of $\mathbb{R}^{2 n}$. By $31, B^{2 n}(1) \subset \Omega=$ $\bar{e}\left(\Omega_{\mathcal{M}}\right)$ and hence $\bar{e}(\pi)=T_{p} S^{2 n-1}$. By applying the same argument to any tripotent $c_{\theta}:=e^{i \theta} \cdot c$, we see that $\Omega$ is contained in the cylinder $\tilde{Z}$ bounded by the envelope of the family of real hyperplanes $\left\{T_{p_{\theta}} S^{2 n-1}, p_{\theta}=\bar{e}\left(c_{\theta}\right)\right\}_{\theta \in \mathbb{R}}$. Let $W \in U(n)$ such that

$$
W \cdot p=\left(z_{1}, 0, \ldots, 0\right)
$$

for some $z_{1} \in \mathbb{C},\left\|z_{1}\right\|=1$. It follows that $W \cdot \tilde{Z}=Z^{2 n}(1)$ and the desired symplectic embedding of $\left(\Omega, \omega_{0}\right)$ into $\left(Z^{2 n}(1), \omega_{0}\right)$ is given by

$$
\Omega \subset \tilde{Z} \stackrel{W}{\rightarrow} Z^{2 n}(1) .
$$


Remark 15. In [36] a similar (symplectic) embedding $\left(\Omega, \omega_{0}\right) \hookrightarrow\left(Z^{2 n}(1), \omega_{0}\right)$ has been considered for the classical Cartan domains.

\subsection{Minimal symplectic atlases of HSSCT}

Consider a closed symplectic manifold $(M, \omega)$. In 44] Yu. B. Rudyak and F. Schlenk have introduced the symplectic Lustermik-Schnirelmann category $S(M, \omega)$, defined as

$$
S(M, \omega)=\min \left\{k \mid M=\mathcal{U}_{1} \cup \cdots \cup \mathcal{U}_{k}\right\}
$$

where each $\mathcal{U}_{i}$ is the image $\Phi_{i}\left(U_{i}\right)$ of a symplectic embedding $\Phi_{i}: U_{i} \rightarrow \mathcal{U}_{i} \subset$ $M$ of a bounded subset $U_{i}$ of $\left(\mathbb{R}^{2 n}, \omega_{0}\right)$ diffeomorphic to an open ball in $\mathbb{R}^{2 n}$. From our results one obtains the upper bound

$$
S\left(M, \omega_{F S}\right) \leq N+1
$$

for Lustermik-Schnirelmann category of a Hermitian symmetric space of compact type $\left(M, \omega_{F S}\right)$, where $N$ is the dimension of the complex projective space $\mathbb{C} P^{N}$ where the manifold can be Kähler embedded via the Borel-Weil embedding $B W: M \rightarrow \mathbb{C} P^{N}$ (see (27)). Indeed, as in the case of the complex Grassmannian $G(k, n)$ (where the Borel-Weil embedding is given by the Plücker embedding $P: G(k, n) \rightarrow \mathbb{C P}^{\left(\begin{array}{l}n \\ k\end{array}\right)-1}$ ), one can define a canonical atlas on $\left(M, \omega_{F S}\right)$ using the $N+1$ holomorphic charts $\Omega_{0}, \ldots, \Omega_{N}$ defined as $\Omega_{j}=M \backslash\left\{B W^{-1}\left(Z_{j}=0\right)\right\}$, and $Z_{j}=0, j=0, \ldots, N$, is the standard hyperplane of $\mathbb{C P}^{N}$. Each $\Omega_{j} \subset \mathbb{C}^{n}, j=0, \ldots, N$, is biholomorphic to the noncompact dual $\Omega$ of $M$. It follows by $(28)$ that $\left(\Omega_{j}, \omega_{0}\right)$ can be symplectically embedded into $\left(M, \omega_{F S}\right)$ for $j=1, \ldots, N$. On the other hand, each $\Omega$ is a bounded domain diffeomorphic to the ball in $\mathbb{R}^{2 n}$ and so $(33$ follows. Our knowledge of the Gromov width of any $\operatorname{HSSCT}\left(M, \omega_{F S}\right)$ can be used to estimate and compute the minimal numbers of Darboux charts needed to cover $M$. This number, introduced in [44] and denoted there by $S_{B}(M, \omega)$, has been computed and estimated for various symplectic manifolds including the complex Grassmannian (see [44, Corollary 5.10]). Using the results of this section, similar computations and related problems (which will appear in a forthcoming paper) can be done for all HSSCT.

\section{The proofs of Theorems 1, 2, 3, 4 and 5}

The following lemma is the key ingredient to achieve the upper bound of Gromov width in Theorems 1, 2 and 3. 
Lemma 16. Let $\left(M, \omega_{F S}\right)$ be an irreducible HSSCT of complex dimension $n$. Then there exist $\alpha\left(M, \omega_{F S}\right)$ and $\beta\left(M, \omega_{F S}\right)$ in $H_{*}(M, \mathbb{Z})$ such that

$$
\operatorname{dim} \alpha\left(M, \omega_{F S}\right)+\operatorname{dim} \beta\left(M, \omega_{F S}\right)=4 n-2 c_{1}(A)
$$

and

$$
\Psi_{A, 0,3}\left(p t ; \alpha\left(M, \omega_{F S}\right), \beta\left(M, \omega_{F S}\right), p t\right) \neq 0 .
$$

Proof. Since the canonical symplectic form $\omega_{F S}$ is Kähler-Einstein, it follows that $\left(M, \omega_{F S}\right)$ is monotone, so that Lemma 10 applies under our assumptions. We need then to show the existence, for every irreducible HSSCT, of a non-vanishing Gromov-Witten invariant $\Psi_{A, 0,3}\left(\alpha\left(M, \omega_{F S}\right), \beta\left(M, \omega_{F S}\right), p t\right)$. This follows from the results about the quantum cohomology of these spaces proved in [1], [8], 27], 42], 49]. Let us recall that the quantum cohomology ring of $M$ is the product $H_{*}(M) \otimes \mathbb{Z}[q]$ endowed with the quantum cup product, defined for any two homology classes $\alpha, \beta \in H_{*}(M)$ as

$$
\alpha * \beta=\sum_{\gamma, d} \Psi_{d A, 0,3}(\alpha, \beta, \gamma) \gamma^{*} q^{d}
$$

the sum running over $d \in \mathbb{Z}$ and $\gamma$ such that $\operatorname{dim}(\alpha)+\operatorname{dim}(\beta)+\operatorname{dim}(\gamma)=$ $4 n-2 d c_{1}(A)$, where $\gamma^{*}$ denotes the dual class of $\gamma$.

Looking at the formulas for the quantum product proved in the abovementioned references, it is not hard to find a Gromov-Witten invariant $\Psi_{A, 0,3}(\alpha, \beta, p t)$ which does not vanish for some classes $\alpha, \beta$. In more details, when $M$ is the Grassmannian $G(k, n)$, by [49] there exist $\alpha \in H_{2 k(n-1)}(M)$ and $\beta \in H_{2 n(k-1)}(M)$ such that this holds; by [42] the same is true for suitable $\alpha=\beta \in H_{(n-1)(n-2)}(S O(2 n) / U(n))$; by Corollary 8 in [27] $\alpha$ and $\beta$ can be taken of codimension $n$ and 1 when $M$ is the Lagrangian Grassmannian $L G(n, 2 n)$; in [8] (see the formulas in Sections 5.1 and 5.2) it is shown that for the Cayley plane (resp. for the Freudenthal variety) one can take for example $\alpha$ and $\beta$ of codimensions 8 and 4, (resp. of codimensions 13 and 5). Finally, in [1] is studied the quantum cohomology of complete intersections, which in particular gives a non-vanishing Gromov-Witten invariant for the complex quadric.

Remark 17. Formulas for quantum products in the homogeneous spaces, expressed in terms of the combinatorial invariants of the Lie algebra of the symmetry group of the space (Dynkin diagram and Weyl group), can be 
found in [16] (see also [15], [14]) and could be also used to prove the above Lemma.

We are now in the position to prove Theorem 1 .

Proof of Theorem 1. In order to use Lemma 9 we can assume, without loss of generality, that $\operatorname{dim} M \geq 4$. Indeed the only irreducible HSSCT of dimension $<4$ is $\left(\mathbb{C P}^{1}, \omega_{F S}\right)$ whose Gromov width is well-known to be equal to $\pi$. Let $A=\left[\mathbb{C} P^{1}\right]$ be the generator of $H_{2}(M, \mathbb{Z})$ as in the Notation at page 1050 . Then the value $\omega_{F S}(A)=\pi$ is clearly the infimum of the $\omega_{F S}$-areas $\omega_{F S}(B)$ of the homology classes $B \in H_{2}(M, \mathbb{Z})$ for which $\omega_{F S}(B)>0$.

By Lemma 16 we have $\Psi_{A, 0,3}(p t ; p t, \alpha, \beta) \neq 0$, with $\alpha=\alpha\left(M, \omega_{F S}\right)$ and $\beta=$ $\beta\left(M, \omega_{F S}\right)$, and hence, by definition of $G W_{g}$,

$$
G W\left(M, \omega_{F S} ; p t, \gamma\right)=G W_{0}\left(M, \omega_{F S} ; p t, \gamma\right)=\pi
$$

with $\gamma=\alpha\left(M, \omega_{F S}\right)$ or $\gamma=\beta\left(M, \omega_{F S}\right)$. It follows by the inequalities (17), (20), (22) and (23) that

$$
c_{G}\left(M, \omega_{F S}\right) \leq C_{H Z}^{(2)}\left(M, \omega_{F S} ; p t, \gamma\right) \leq C_{H Z}^{(2 o)}\left(M, \omega_{F S} ; p t, \gamma\right) \leq \pi
$$

with $\gamma=\alpha\left(M, \omega_{F S}\right)$ or $\gamma=\beta\left(M, \omega_{F S}\right)$. Combining this with the lower bound $c_{G}\left(M, \omega_{F S}\right) \geq \pi$ coming from the inclusion $B^{2 n}(1) \subset\left(\Omega, \omega_{0}\right)$ (cfr. (31)), the symplectic embedding $\Phi_{\Omega}:\left(\Omega, \omega_{0}\right) \rightarrow\left(M, \omega_{F S}\right)$ (cfr. (28)) and the monotonicity and nontriviality of $c_{G}$, one gets:

$$
c_{G}\left(M, \omega_{F S}\right)=C_{H Z}^{(2)}\left(M, \omega_{F S} ; p t, \gamma\right)=C_{H Z}^{(2 o)}\left(M, \omega_{F S} ; p t, \gamma\right)=\pi
$$

with $\gamma=\alpha\left(M, \omega_{F S}\right)$ or $\gamma=\beta\left(M, \omega_{F S}\right)$. This concludes the proof of Theorem 1 .

Remark 18. Observe that we have proven more than stated in Theorem 1 . Indeed, we have computed the value of Lu's pseudo symplectic capacities evaluated at the homology class of a point and at $\alpha\left(M, \omega_{F S}\right)\left(\right.$ or $\left.\beta\left(M, \omega_{F S}\right)\right)$, namely

$$
\begin{aligned}
c_{G}(M, \omega) & =C_{H Z}^{(2)}\left(M, \omega ; p t, \alpha\left(M, \omega_{F S}\right)\right)=C_{H Z}^{(2 o)}\left(M, \omega ; p t, \alpha\left(M, \omega_{F S}\right)\right) \\
& =C_{H Z}^{(2)}\left(M, \omega ; p t, \beta\left(M, \omega_{F S}\right)\right)=C_{H Z}^{(2 o)}\left(M, \omega ; p t, \beta\left(M, \omega_{F S}\right)\right)=\pi .
\end{aligned}
$$

This extends the result obtained by G. Lu for the complex Grassmannian (cfr. [35, Theorem 1.15] for details) to HSSCT. 
Remark 19. An alternative proof of the upper bound $c_{G}\left(M, \omega_{F S}\right) \leq \pi$ in Theorem 1 can be achieved by combining Lemma 16 with [23, Proposition 4.1 ] which asserts that if $(M, \omega)$ is a symplectic manifold of (real) dimension $2 n$, $B \in H_{2}(M, \mathbb{Z})$ is an indecomposable spherical class and $\Phi_{B, 0,3}\left(p t, \alpha_{0}, \beta_{0}\right) \neq$ 0 , for suitable $\alpha_{0}$ and $\beta_{0}$ in $H_{*}(M, \mathbb{Z})$ (which necessarily satisfy $\operatorname{dim} \alpha_{0}+$ $\left.\operatorname{dim} \beta_{0}=4 n-2 c_{1}(B)\right)$ then $c_{G}(M, \omega) \leq \omega(B)$.

Actually, the GW invariant $\Phi_{B, 0,3}\left(p t, \alpha_{0}, \beta_{0}\right) \neq 0$ for some $B \in H_{2}(M, \mathbb{Z})$ implies that there exists a rational curve of class $B$ through a generic point in $M$ and hence the inequality $c_{G}(M, \omega) \leq \omega(B)$ by the Gromov's arguments in [17]; see [23], [7] for details.

In order to prove Theorem 2 we need the following lemma, interesting on its own sake, which extends Lu's formula (20) in [35, Theorem 1.16] (for the Grassmannian) to the case of HSSCT.

Lemma 20. Let $\left(M, \omega_{F S}\right)$ be a HSSCT and let $(N, \omega)$ be any closed symplectic manifold. Then

$$
C_{H Z}^{(2 o)}\left(N \times M, \omega \oplus a \omega_{F S} ; p t,[N] \times \gamma\right) \leq|a| \pi
$$

for any $a \in \mathbb{R} \backslash\{0\}$ and $\gamma=\alpha\left(M, \omega_{F S}\right)$ or $\gamma=\beta\left(M, \omega_{F S}\right)$, with $\alpha\left(M, \omega_{F S}\right)$ and $\beta\left(M, \omega_{F S}\right)$ given by Lemma 16 .

Proof. Since by 34 we have $\Psi_{A, 0,3}^{M}(p t ; \alpha, \beta, p t) \neq 0$, with $\alpha=\alpha\left(M, \omega_{F S}\right)$ and $\beta=\beta\left(M, \omega_{F S}\right)$, it follows by Lemma 11 that

$$
\Psi_{B, 0,3}^{N \times M}\left(p t ;[N] \times \alpha\left(M, \omega_{F S}\right),[N] \times \beta\left(M, \omega_{F S}\right), p t\right) \neq 0
$$

for $B=0 \times A$, where 0 denotes the zero class in $H_{2}(N, \mathbb{Z})$. Hence (39) easily follows from 23 in Lemma 9.

Proof of Theorem 2. To see (5) we assume $r>1$ because of the result in Theorem 1. It immediately follows from (17) and 20) in Lemma 7 and by (39) that

$$
c_{G}\left(M_{1} \times \cdots \times M_{r}, \omega_{F S}^{1} \oplus \cdots \oplus \omega_{F S}^{r}\right) \leq \pi .
$$

On the other hand, we have the symplectic embeddings

$$
\times_{j=1}^{r} B^{2 n_{j}}(1) \subset \times_{j=1}^{r} \Omega_{j} \stackrel{\Phi_{\Omega_{1}} \times \cdots \times \Phi_{\Omega_{r}}}{\longrightarrow} \times_{j=1}^{r} M_{j}
$$


(induced by (31) and (28) respectively) and the natural inclusion

$$
B^{2 n_{1}+\cdots+2 n_{r}}(1) \subset \times_{j=1}^{r} B^{2 n_{j}}(1) .
$$

Thus, it follows by the monotonicity and nontriviality of $c_{G}$ that

$$
c_{G}\left(M_{1} \times \cdots \times M_{r}, \omega_{F S}^{1} \oplus \cdots \oplus \omega_{F S}^{r}\right) \geq \pi
$$

Hence (5) follows. As we have already pointed out in the Introduction, inequality (6) is a straightforward consequence of (8) in Theorem 3 .

Inequality (7) follows by (4), by the monotonicity of $c_{H Z}$ and from the fact that for two compact symplectic manifolds $\left(N_{1}, \omega_{1}\right)$ and $\left(N_{2}, \omega_{2}\right)$

$$
c_{H Z}\left(N_{1} \times N_{2}, \omega_{1} \oplus \omega_{2}\right) \geq c_{H Z}\left(N_{1}, \omega_{1}\right)+c_{H Z}\left(N_{2}, \omega_{2}\right)
$$

(see [35, Lemma 4.3, p. 43] for a proof). This concludes the proof of Theorem 2.

Remark 21. The upper bound

$$
c_{G}\left(M_{1} \times \cdots \times M_{r}, \omega_{F S}^{1} \oplus \cdots \oplus \omega_{F S}^{r}\right) \leq \pi
$$

obtained in the proof of Theorem 2 can also be achieved by using the fact that HSSCT and their products are uniruled manifolds (see Definition 1.14, Theorem 1.27 in [35] and the remark following this theorem).

Remark 22. Note that another interesting result shown in 35, Theorem 1.16] is formula (21) in [35]. One can prove the analogous of this formula using the techniques developed so far. That is

$$
C_{H Z}^{(2 o)}\left(\times_{j=1}^{r} M_{j}, \oplus_{j=1}^{r} a_{j} \omega_{F S}^{j} ; p t, \times_{j=1}^{r} \alpha_{j}\right) \leq\left(\left|a_{1}\right|+\cdots+\left|a_{r}\right|\right) \pi
$$

for all $a_{j} \in \mathbb{R} \backslash\{0\}$ and $\alpha_{j}=\alpha_{j}\left(M_{j}, \omega_{F S}^{j}\right)$ or $\beta_{j}=\beta_{j}\left(M_{j}, \omega_{F S}^{j}\right)$.

Remark 23. We do not know if the inequality

$$
c_{H Z}\left(M_{1} \times \cdots \times M_{r}, a_{1} \omega_{F S}^{1} \oplus \cdots \oplus a_{r} \omega_{F S}^{r}\right) \leq\left(\left|a_{1}\right|+\cdots+\left|a_{r}\right|\right) \pi .
$$

holds true. Unfortunately, the proof given by Lu in the case of product of projective spaces [35, Theorem 1.21] does not extend to the general case of HSSCT. Indeed the Gromov-Witten invariant $\Psi_{B, 0, m+2}\left(p t, p t, \beta_{1}, \ldots, \beta_{m}\right)$ of $M=M_{1} \times \cdots \times M_{r}$ does not vanish (for some homology classes $\beta_{1}, \ldots, \beta_{m}$ ) 
if and only if all the $M_{j}$ 's are projective spaces, since it is easily checked that the dimension condition $\sum_{j=1}^{m} \operatorname{deg}\left(\beta_{j}\right)=2\left(c_{1}(B)-\operatorname{dim}(M)-1+m\right)$, necessary for the Gromov-Witten invariant to be nonzero ([39], p. 11), is satisfied only in this case. For comments and conjectures related to this problem the reader is referred to [35, Corollary 1.19 and Example 1.20]).

Proof of Theorem 3. From (17) and (20) in Lemma 7 and by (39) it follows that

$$
c_{G}\left(N \times M, \omega \oplus a \omega_{F S}\right) \leq C_{H Z}^{(2 o)}\left(N \times M, \omega \oplus a \omega_{F S} ; p t,[N] \times \gamma\right) \leq|a| \pi,
$$

where $\gamma=\alpha\left(M, \omega_{F S}\right)$ (or $\left.\gamma=\beta\left(M, \omega_{F S}\right)\right)$, which yields the desired inequality (8).

Proof of Theorem 4. By

$$
\left(B^{2 n}(1), \omega_{0}\right) \subset\left(\Omega, \omega_{0}\right) \stackrel{W}{\rightarrow}\left(Z^{2 n}(1), \omega_{0}\right),
$$

(given by (31) and (32) respectively) and the monotonicity and nontriviality of $c_{G}$ and $c_{H Z}$ we get $c_{G}\left(\Omega, \omega_{0}\right)=c_{H Z}\left(\Omega, \omega_{0}\right)=\pi$, namely (12) and (13). Analogously, let us denote $M_{j}$ the compact dual of $\Omega_{j}$ : by (6) and by the symplectic embedding

$$
\left(\times_{j=1}^{r} \Omega_{j}, \oplus_{j=1}^{r} a_{j} \omega_{0}^{j}\right) \stackrel{\Phi_{\Omega_{1}} \times \cdots \times \Phi_{\Omega_{r}}}{\longrightarrow}\left(\times_{j=1}^{r} M_{j}, \oplus_{j=1}^{r} a_{j} \omega_{F S}^{j}\right)
$$

induced by (28) one obtains (15) which, together with the symplectic embed$\operatorname{ding} \times_{j=1}^{r} B^{2 n_{j}}(1) \subset \times_{j=1}^{r} \Omega_{j}$ (induced by $(31)$ ) and $(40)$ yields 14 .

In order to prove Theorem 5 we need the following interesting result of Lu.

Lemma 24. Let $(N, \omega)$ be any closed symplectic manifold. Then, for any $r>0$ one has

$$
c_{H Z}\left(N \times B^{2 n}(r), \omega \oplus \omega_{0}\right)=c_{H Z}\left(N \times Z^{2 n}(r), \omega \oplus \omega_{0}\right)=\pi r^{2} .
$$

where $Z^{2 n}(r)$ is given by (3).

Proof. See [35, Theorem 1.17, p.14]. 
Proof of Theorem 5. By $\left(B^{2 n}(1), \omega_{0}\right) \subset\left(\Omega, \omega_{0}\right) \stackrel{W}{\rightarrow}\left(Z^{2 n}(1), \omega_{0}\right)$ one has the embeddings

$$
\left(N \times B^{2 n}(1), \omega \oplus \omega_{0}\right) \subset\left(N \times \Omega, \omega \oplus \omega_{0}\right) \stackrel{i d_{N} \times W}{\rightarrow}\left(N \times Z^{2 n}(1), \omega \oplus \omega_{0}\right)
$$

and so the desired (16), i.e. $c_{H Z}\left(N \times \Omega, \omega \oplus \omega_{0}\right)=\pi$, follows by Lemma 24 and the monotonicity of $c_{H Z}$.

\section{Final remarks on Seshadri constants}

Our knowledge of the Gromov width of a HSSCT allows us to obtain an upper bound of the Seshadri constant of an ample line bundle over a HSSCT $\left(M, \omega_{F S}\right)$. Recall that given a compact complex manifold $(N, J)$ and a holomorphic line bundle $L \rightarrow N$ the Seshadri constant of $L$ at a point $x \in N$ is defined as the nonnegative real number

$$
\epsilon(L, x)=\inf _{C \ni x} \frac{\int_{C} c_{1}(L)}{\operatorname{mult}_{x} C},
$$

where the infimum is taken over all irreducible holomorphic curves $C$ passing through the point $x$ and mult $_{x} C$ is the multiplicity of $C$ at $x$ (see [10] for details). The (global) Seshadri constant is defined by

$$
\epsilon(L)=\inf _{x \in N} \epsilon(L, x)
$$

Note that Seshadri's criterion for ampleness says that $L$ is ample if and only if $\epsilon(L)>0$. P. Biran and K. Cieliebak [6, Prop. 6.2.1] have shown that

$$
\epsilon(L) \leq c_{G}\left(N, \omega_{L}\right)
$$

where $\omega_{L}$ is any Kähler form which represents the first Chern class of $L$, i.e. $c_{1}(L)=\left[\omega_{L}\right]$. Consider now an irreducible $\operatorname{HSSCT}\left(M, \omega_{F S}\right)$ and the line bundle $L \rightarrow M$ such that $c_{1}(L)=\left[\frac{\omega_{F S}}{\pi}\right]$ ( $L$ can be taken as the pull-back via the Borel-Weil embedding (27) of the universal bundle of $\mathbb{C P}^{N}$ ). Therefore, by using the upper bound $c_{G}\left(M, \omega_{F S}\right) \leq \pi$ and the conformality of $c_{G}$ we get:

Corollary 25. Let $\left(M, \omega_{F S}\right)$ be an irreducible HSSCT and let $L \rightarrow M$ as above. Then $\epsilon(L) \leq 1$. 


\section{References}

[1] A. Beauville, Quantum cohomology of complete intersections. Mat. Fiz. Anal. Geom., 2 (1995), no. 3-4, 384-398.

[2] W. Bertram, The geometry of Jordan and Lie structures. Lecture Notes in Mathematics 1754, Springer-Verlag (2000).

[3] P. Biran, Symplectic packing in dimension 4. Geom. Funct. Anal., 7 (1997), 420-437.

[4] P. Biran, A stability property of symplectic packing. Invent. Math., 136 (1999) 123-155.

[5] P. Biran, From symplectic packing to algebraic geometry and back. European Congress of Mathematics, Vol. II (Barcelona, 2000); Progr. Math., 202, Birkhauser, Basel (2001), 507-524.

[6] P. Biran and K. Cieliebak, Symplectic topology on subcritical manifolds. Commentarii Mathematici Helvetici, 76 (2001), 712-753.

[7] A. C. Castro, Upper bound for the Gromov width of coadjoint orbits of type A. arXiv:1301.0158v1.

[8] P. E. Chaput, L. Manivel and N. Perrin, Quantum cohomology of minuscule homogeneous spaces. Transform. Groups, 13 (2008), no. 1, 47-89.

[9] F. Cuccu and A. Loi, Global symplectic coordinates on complex domains. J. Geom. Phys., 56 (2006), 247-259.

[10] J. P. Demailly, $L^{2}$-vanishing theorems for positive line bundles and adjunction theory. In: Trascendental methods in Algebraic Geometry (F. Catanese and C. Ciliberto, eds.), Lecture Notes in Mathematics 1646, Springer-Verlag, Berlin, 1992, pp. 1-97.

[11] A. J. Di Scala and A. Loi, Symplectic duality of symmetric spaces. Advances in Mathematics, 217 (2008), 2336-2352.

[12] A. J. Di Scala, A. Loi and G. Roos, The bisymplectomorphism group of a bounded symmetric domain. Transform. Groups, 13 (2008), no. 2, 283-304.

[13] A. J. Di Scala, A. Loi and F. Zuddas, Symplectic duality between complex domains. Monatsh. Math., 160 (2010), 403-428.

[14] W. Fulton, On the quantum cohomology of homogeneous varieties. In: The Legacy of Niels Henrik Abel, Springer, Berlin, 2004, pp. 729-736. 
[15] W. Fulton and R. Pandharipande, Notes on stable maps and quantum cohomology. In: Algebraic Geometry (Santa Cruz, 1995), Proc. Sympos. Pure Math., Vol. 62, Part 2, American Mathematical Society, Providence, RI, 1997, pp. 45-96.

[16] W. Fulton and C. Woodward, On the quantum product of Schubert classes. J. Algebraic Geom., 13 (2004), no. 4, 641-661.

[17] M. Gromov, Pseudoholomorphic curves in symplectic manifolds. Invent. Math., 82 (1985), no. 2, 307-347, Springer-Verlag (1986).

[18] S. Helgason, Differential geometry, Lie groups, and symmetric spaces. Graduate Studies in Mathematics, 34, American Mathematical Society, Providence, RI, 2001.

[19] H. Hofer and C. Viterbo, The Weinstein conjecture in the presence of holomorphic spheres. Communications on Pure and Applied Mathematics, 45 (1992), 583-622.

[20] H. Hofer and E. Zehnder, A new capacity for symplectic manifolds. In: Analysis et cetera (P. Rabinowitz and E. Zehnder, eds.), Academic Press, New York, 1990, pp. 405-429.

[21] H. Hofer and E. Zehnder, Symplectic invariants and Hamiltonian Dynamics. Birkhäuser, Basel 1994.

[22] M.-Y. Jiang, Symplectic embeddings from $\mathbb{R}^{2 n}$ into some manifolds. Proc. Roy. Soc. Edinburgh Sect. A, 130 (2000), 53-61.

[23] Y. Karshon and S. Tolman, The Gromov width of complex Grassmannians. Algebr. Geom. Topol., 5 (2005), 911-922.

[24] S. Kobayashi, Hyperbolic manifolds and holomorphic mappings. An introduction. Second edition, World Scientific Publishing Co. Pte. Ltd., Hackensack, NJ, 2005.

[25] M. Koecher, The Minnesota Notes on Jordan Algebras and Their Applications. Lecture Notes in Mathematics 1710, Springer-Verlag (1999).

[26] M. Koecher, An elementary approach to Bounded Symmetric Domains. Rice University (1969).

[27] A. Kresch and H. Tamvakis, Quantum cohomology of the Lagrangian Grassmannian. J. Algebraic Geom., 12 (2003), no. 4, 777-810.

[28] J. Latschev, D. McDuff and F. Schlenk, The Gromov width of 4dimensional tori. Preprint (2011), arXiv:1111.6566. 
[29] A. Loi and R. Mossa, The diastatic exponential of a symmetric space. Math. Z., 268 (2011), no. 3-4, 1057-1068.

[30] A. Loi and M. Zedda, Calabi's inhomogeneous Einstein manifold is globally symplectomorphic to $\mathbb{R}^{2 n}$. Diff. Geom. and its App., 30 (2012), $145-147$.

[31] A. Loi and M. Zedda, Global symplectic coordinates on gradient KählerRicci solitons. To appear in Monatsh. Math.

[32] A. Loi and F. Zuddas, Symplectic maps of complex domains into complex space forms. J. Geom. Phys., 58 (2008), 888-899.

[33] O. Loos, Bounded Symmetric Domains and Jordan pairs. Lecture Notes, Irvine (1977).

[34] G. Lu, Weinstein conjecture on some symplectic manifolds containing the holomorphic spheres. Kyushu J. Math., 52 (1998), 331-351.

[35] G. Lu, Gromov-Witten invariants and pseudo symplectic capacities. Israel J. Math., 156 (2006), 1-63.

[36] G. Lu, H. Ding and Q. Zhang, Symplectic capacities of classical domains. Int. Math. Forum, 2 (2007), no. 25-28, 1311-1317.

[37] D. McDuff, Blowups and symplectic embeddings in dimension 4. Topology, 30 (1991), 409-421.

[38] D. McDuff and L. Polterovich, Symplectic packings and algebraic geometry. Invent. math., 115 (1994), 405-429.

[39] D. McDuff and D. Salamon, J-holomorphic curves and quantum cohomology. University Lecture Series 6, American Mathematical Society, Providence, RI, 1994.

[40] D. McDuff and D. Salamon, Introduction to symplectic Topology. 2nd edition, Oxford University Press, Oxford, 1998.

[41] R. Mossa, The volume entropy of local Hermitian symmetric space of noncompact type. Diff. Geom. Appl., 31 (2013) , no. 5, 594-601.

[42] Y. Nishimori and Y. Ohnita, Quantum cohomology ring for Hermitian symmetric spaces of type DIII. Topics in applied and theoretical mathematics and computer science, 232-237, Math. Comput. Sci. Eng., WSEAS, Athens, 2001. 
[43] G. Roos, Jordan triple systems. In: Analysis and Geometry on Complex Homogeneous Domains (J. Faraut, S. Kaneyuki, A. Korányi, Q.k. Lu and G. Roos, eds.), pp. 425-534, Progress in Mathematics, Vol. 185, Birkhäuser, Boston, 2000.

[44] Y. B. Rudyak and F. Schlenk, Minimal atlases of closed symplectic manifolds. Commun. Contemp. Math., 9 (2007), no. 6, 811-855.

[45] I. Satake, Algebraic structures of symmetric domains. Publications of the Mathematical Society of Japan 14, Kano Memorial Lectures 4, Iwanami Shoten Pub. and Princeton University Press (1980).

[46] F. Schlenk, Embedding problems in symplectic geometry. de Gruyter Expositions in Mathematics 40, Walter de Gruyter Verlag, Berlin, 2005.

[47] M. Schwarz, On the action spectrum for closed symplectically aspherical manifolds. Pacific Journal of Mathematics, 193 (2000), 419-461.

[48] K. Siburg, Symplectic capacities in two dimension. Manuscripta Mathematica, 78 (1993), 149-163.

[49] B. Siebert and G. Tian, On quantum cohomology rings of Fano manifolds and a formula of Vafa and Intriligator. Asian J. Math., 1 (1997), 679-695.

[50] M. Takeuchi, Homogeneous Kähler Manifolds in Complex Projective Space. Japan J. Math., 4 (1978), 171-219.

[51] M. Zoghi, The Gromov Width of Coadjoint Orbits of Compact Lie Groups. Thesis (Ph.D.) University of Toronto (Canada), 2010.

Dipartimento di Matematica e Informatica, Università di Cagliari

Via Ospedale 72-09124 CAgliari, Italy

E-mail address: loi@unica.it

Dipartimento di Matematica, Università di Cagliari

Via Ospedale 72-09124 CAGLiari, Italy

E-mail address: roberto.mossa@gmail.com

Dipartimento di Matematica e Informatica

Via Delle Scienze 206, Udine, ItAly

E-mail address: fabio.zuddas@uniud.it

ReCEIVED April 26, 2013 
